\title{
Emerging Evidence for Newer Approaches to Sports \& Exercise: Tools for Man Making \& Nation Building
}

\author{
Dr Pavankumar kohli, Dr Sunil Nadkarni, Dr Satishchandra Gore, Bhagyashree Kulkarni, \\ Dr Nabh Patil \\ ${ }^{I}$ Consultant Sports Orthopedic Surgeon, B.K.L. Walawalkar Hospital, Dervan, India \\ ${ }^{2}$ Consultant Spine Surgeon, B.K.L. Walawalkar Hospital, Dervan, India \\ ${ }^{3}$ Consultant Spine Surgeon, B.K.L. Walawalkar Hospital, Derwan, India \\ ${ }^{4}$ Research Assistant, Department of sports \& orthopedic Surgery, B.K.L. Walawalkar Hospital, Dervan, India \\ ${ }^{5}$ Research Assistant, Department of sports \& orthopedic Surgery, B.K.L. Walawalkar Hospital, Dervan, India
}

\section{Introduction}

All over the world and for time immemorial man has been trying to find ways to improve his quality of life. Sedentary life style is hall mark of 21 century. Most of the maladies affecting comfort seeking man can be relieved by physical activity and exercises. We have created a conceptual framework for life style modification and exercise intervention for rural and semi urban population at Sports medicine center Shree Kshetra Dervan. Our work will be published in 3 parts

1. Concept framework and evidence base from best publications pooled from sources as first paper ( present paper )

2. Action taken on identified area for intervention as second paper.

3. Outcomes \& results of the interventions mainly inform of exercises \& development of new exercises.

\section{Materials And Methods}

32 international published studies on benefits of Sports were reviewed. Journals from America, Canada, UK and Ireland, Australia and Asia were studied and all papers were selected for the final reviewed list based on applicability, study design, method of study and date of publishing. They were then grouped under five different headings of Physical, Mental, Emotional, Economical and Social impact. The relevant findings were then rearranged for this publication and the first two important papers presented (due to space constraints of this annual conference) according to significance and validation.

\section{Results}

The principal studies with the main headings are presented below.

\section{Physical}

Warburton et.al. in 2006 presented a work on Health benefits of Physical activity. The authors confirm that there is irrefutable evidence in the primary and secondary prevention of many chronic diseases chiefly Cardiac diseases, Diabetes, Cancer, Hypertension, Obesity, Depression and Osteoporosis. Compelling evidence shows that regular sports activity decreases chances of sudden premature death in both sexes. Regular exercise also decreases the risk of Breast, Colon and ovarian cancers. Other effects like better body composition, enhanced lipoprotein profiles, glucose homeostasis, and insulin sensitivity, reduced blood pressure, decreased coagulation and improved coronary blood flow and enhanced endothelial function. The authors state that there appears to be a linear relationship between physical activity and health status with greatest improvements occurring in people who move from unfit to fit! [1]

Europe heart network on physical activity stated in 1999. A sedentary lifestyle more than doubles the risk of CVD and it is possible to reduce the risk of coronary heart disease by making lifestyle changes even relatively late in life. Physical activity influences CVD risk in a number of ways: (i) Lowers heart rate. (ii) Raises insulin sensitivity. (iii) Lowers blood pressure. (iv) Raises HDL/total blood cholesterol ratio. (v) Helps with weight control. The document provides a summary of the evidence regarding required levels and frequency of activity. These are: (i) Daily, moderate and accumulated (30 minutes per day). (ii) Frequency is more important than intensity (although exercise to increase cardio-respiratory fitness has to be vigorous and tends to be achieved through dedicated sports or exercise activities). (iii) Intensity depends on existing levels of fitness. Common forms of moderate-intensity activities for the moderately fit include brisk walking at $5-6 \mathrm{~km} / \mathrm{h}$, cycling at approximately $16 \mathrm{~km} / \mathrm{h}$, walking up stairs or heavy forms of gardening and housework. (iv) Accumulated activity is also effective and activity needs to be current and continuing. On this basis it recommends: (i) Adults should accumulate 30 minutes of moderate-intensity physical activity most and preferably every day of the 
week. (ii) All young people (5-18) should participate in physical activity of at least moderate intensity for one hour per day. $[2,3]$

Other important studies prove a protective role of sustained fitness levels on breast cancer (Californian teachers study of more than 100,000 teachers, 2007, USA) proved that women who exercised daily had substantially lower risk of developing breast cancer than sedentary women. Welsh et.al from Australia in 2005 in study of Physical conditioning/Sports and effect on control of Asthma, conclusively proves the benefits of Sports and exercise in decreasing the attacks, intensity, medications and hospitalizations of Asthma. The authors recommended regular sports/physical activity for associated general health benefits and improvement of asthma management.[4]

\section{Mental}

In 2003 Coe DP and Pivarnik JM study the effects of Physical education on academic achievement in children, the assessment was done on middle school students and it was found that beyond a "Threshold physical activity" contributed highly to better scholastic achievement [5]. The metanalysis report of the relationship between physical activity and cognition in children studied by the Silbey et.al. in 2003[6]. Present the observations of significant improvement by Physical activity and cognition (perception skills, IQ, verbal test, Maths Tests \& Memory). Greatest impact was found in elementary and middle school students and the authors strongly protested the cutting down of hours used in Physical education. Other valuable studies show decrease in disruptive behaviours and increase in desirable behaviours and attention span of children.[7,8,9]

\section{Emotional}

Page et.al. in 1998 present a study on a School sports as a protection factor against adolescent risk behaviours in which Male and female students reporting participation in one or two teams were significantly more likely no not have engaged in cigarette smoking, drug use as compared to non sportspersons.[10,11] In the study of Sport Participation \& Health Factors Pyle et.al. and Mcquivey et. al. 2003 studied 770 high school students aged around 16 yrs \& found that both boys and girls competitive sports participation was associated with a lower frequency of mental health problems, eating and dietary problems and total risks compared to non-competitive peers.[11]

In other important studies, Scully \& Kremer's critical review of Physical exercise and psychological well being in 1998 is a masterpiece which offers a critical examination of evidence relating to the relationship between physical exercise and psychological benefits. The review examines existing literature on exercise and mental health in relation to changes in anxiety, depression, mood, self-esteem, and stress reactivity, premenstrual syndrome and body image. The general conclusion is that a range of exercise regimens may be able to play a therapeutic role in relation to a number of psychological disorders [12].With regard to depression the authors conclude that it seems safe to accept that physical exercise regimens will have a positive influence, with the most powerful effects noted among clinical populations. Limited evidence would suggest that aerobic exercise is most effective, including activities such as walking, jogging, cycling, light circuit training and weight training and that regimens which extend over several months appear to yield the most positive results. With regard to anxiety the literature unequivocally supports the positive effects of exercise, with short bursts of exercise appearing to be sufficient. In addition, the nature of the exercise does not appear to be crucial, with the most positive effects being among those who adhere to programs for several months. It would appear that aerobic exercise (of sufficient intensity to elevate the heart rate significantly above resting pulse rate for over 21 minutes) may significantly enhance stress responsively, especially in relation to stress related to lifestyle or work. In respect of mood states both aerobic and anaerobic exercise can be associated with an elevation of mood state, particularly for clinical samples, although it is likely that more than one underlying mechanism may be implicated. With regard to premenstrual syndrome (PMS) the evidence points to the benefits of exercise, with less strenuous forms of non-competitive exercise most effective [12, 13,14,15,16]

Economical

Sports changes image of cities attracts tourists and encourages inward investment. They also provide a case study of Manchester commonwealth Games showing how the Games provided a clear economic boost for the area $[17,18,19,20]$. The economic impact of major sports events, Sports industry research center in 2006 reviews impact of 10 major sports events and concludes that both direct and indirect benefits ensue viz direct from tickets sales, visitors, local development projects, media right etc and indirect from sport development, feel good factor of local population, local image enhancement [21,22, 23].

Other noteworthy publications include Higher medical costs associated with physical inactivity, Consistent and substantial medical cost differences were found in all age groups and sexes on fewer physician visits, lesser hospital stays and less medication and the effect was most pronounced among women of middle age group. Increasing activity levels of sedentary adults could result in medical savings of $300 \$-1053 \$$ per 
person per annum a US saving of 76.6 billion per annum. (Incidentally this is equal to the annual budget of all African states put together.) $[22,23,24]$

\section{Social}

In the study of Measuring the impact of crime reduction interventions involving sports activities for young people, authors concluded that Sports works at primary, secondary \& Tertiary levels and works by a) providing diversion from crime b) deterring criminality c) enables self development \& d) directs towards pro social values [25] Relationship between athletic participation and high school students leadership ability presented by the Dobonz et.al. in 1999 was the major finding, consistent with previous research, was that high school athletes outscored their non-athlete peers on the leadership ability measure. Female athletes showed greater leadership ability than male athletes, although the difference was not statistically significant. The authors conclude that there is a possibility that athletics offers young women and men the chance to improve leadership ability. The authors recommend maintaining athletic programs for the purposes of developing leadership and suggest that educational policy-makers and administrators should re-examine any proposed budget cuts that may affect athletic participation.[26, 27]

Other noteworthy publications are "Sporting capital: changes \& challenges in Victoria "from Australia in 1999 recommended Social capital development via leadership, membership, participation, skill development and community development work of clubs. (ii) Creation of community hubs and key social places. (iii) Environmental and physical development via facilities, spaces and landscape preservation. (iv) Health improvement and promotion. (v) Cultural values via the maintenance of traditions. Although this can reinforce dominant cultural values such as sexism or racism, new ways of working have the potential to challenge this. (vi) Economic development and town survival via sports events and festivals. (vii) Community safety via development of social networks, provision of life-saving services (e.g. learn-to-swim programs). (viii) Community control and investment via a local sense of control. (ix) Junior sports development. (x) Community identity and local pride. They also outline a set of common issues which clubs face: (i) Viability and sustainability (especially regarding volunteers). (ii) Increased regulation of activity. (iii) Managing increased complexity. (iv) Government expectations of rural communities. (v) Finance [28]

\section{Discussion}

One cannot but wonder in face of such proof and scientific material that we will cause most harm to ourselves and our posterity if efforts are not made to encourage sports and physical activity. Our health is in our hands, literally! And the remedy is simple and free, preventive and curative and a single solution for so many problems. May they be from physical, mental, emotional, economic or social spheres? [29-32]

A sport never let's a sportsperson down.

\section{Conclusion}

The way forward for any well intentioned administrator/ trust/ caregiver is to work on prevention rather than cure. Exercise also serves as the best form of treatment when combined with modern treatment for physical, mental, emotional \& social maladies. It also makes the best financial sense to keep exercise as the center of health programmes.

\section{References}

[1]. Warburton DER, Nicol CW, Bredin SSD., Health benefits of physical activity: the evidence. CMAJ., 2006;174:801-9.

[2]. Physical activity and health in Europe: evidence for action edited by Cavill N, Kahlmeier S, Racioppi F, 2006.

[3]. Physical Activity and Cardiovascular Disease Prevention in the European Union 1999. http://www.ehnheart.org/aboutus/overview.html

[4]. Welsh L, Kemp JG, Roberts RG, Effects of physical conditioning on children and adolescents with asthma, Sports Med. 2005;35(2):127-41

[5]. Coe DP, Pivarnik JM, Womack CJ, Reeves MJ, Malina RM. Effect of physical education and activity levels on academic achievement in children, Med Sci Sports Exerc. 2006 Aug;38(8):1515-9.

[6]. Sibley, BA, Etiner, JL, The relationship between physical actively and cognition in children: a meta-analysis. Pediatric Exercise Science, 2003; 15, 243-256.

[7]. Tomporowski PD, Effects of acute bouts of exercise on cognition, Acta Psychol (Amst). 2003 ;112(3):297-324.

[8]. Boone E, Leadbeater B. Game on: Diminishing risks for depressive symptoms in early adolescence through positive involvement in team sports. Journal of Research on Adolescence. 2006;16:79 90. doi:10.1111/j.1532-7795.2006.00122.x.

[9]. Larun L, Nordheim LV, Ekeland E, Hagen KB, Heian F. Cochrane Database of Systematic Reviews. Cochrane AN: 2009. Exercise in prevention and treatment of anxiety and depression among children and young people; p. CD004691.

[10]. Page RM, Hammermeister J, Scanlan A, Gilbert L. Is school sports participation a protective factor against adolescent health risk behaviors? J Health Educ 1998;29:186-192.

[11]. Pyle RP, McQuivey RW, Brassington GS, Steiner H. High school student athletes: associations between intensity of participation and health factors, Clin Pediatr (Phila). 2003;42(8):697-701..

[12]. Scully D, Kremer J, Meade MM, Graham R, Dudgeon K, Physical exercise and psychological well being: a critical review, Br J Sports Med 1998;32:111-120 
[13]. Matz-Costa1 C, Carr DC , McNamara TK, Jacquelyn BJ, Physical, Cognitive, Social, and Emotional Mediators of Activity Involvement and Health in Later Life, Research on Aging 2016; 38(7):791-815.

[14]. Newsom TJ, Shaw BA, August KJ, Strath SJ, Physical activity-related social control and social support in older adults: Cognitive and emotional pathways to physical activity, JHP. 2016; 1-7

[15]. Kok, BE, Fredrickson, BL. Upward spirals of the heart: Autonomic flexibility, as indexed by vagal tone, reciprocally and prospectively predicts positive emotions and social connectedness. Biological Psychology, 2010; 85: 432-436

[16]. Kok BE, Coffey KA, Cohn MA, Catalino LI, Vacharkulksemsuk T, Algoe1 SB, Brantley M, Fredrickson BL, How Positive Emotions Build Physical Health: Perceived Positive Social Connections Account for the Upward Spiral Between Positive Emotions and Vagal Tone, APS.,2013; 24(7): 1123-1132.

[17]. Gratton C, Shibli S, Coleman R, Sport and Economic Regeneration in Cities, Urban Studies, 2005; 42, Nos 5/6, 985- 999.

[18]. Davies LE, Sport and economic regeneration: a winning combination? Sport in Society, SHURA. 2010; $13(10): 1438-1457$.

[19]. Pye PN, Toohey K, Cuskelly G, The social benefits in sport city planning: a conceptual framework, Sport in Society, 2015;18:10, 1199-1221,

[20]. Davies LE, A wider role for sport: community sports hubs and urban regeneration, Sport in Society, http://dx.doi.org/10.1080/17430437.2016.1159192

[21]. Gratton C, Shibli S, Coleman S, The economic impact of major sports events: a review of ten events in the UK, The economic impact of major sports events, 2006: 41-58

[22]. Li S, Evaluating economic impacts of major sports events - a meta analysis of the key trends, Current Issues in Tourism, 2011; 591-611.

[23]. Study on the Contribution of Sport to Economic Growth and Employment in the EU, Study commissioned by the European Commission, Directorate-General Education and Culture Final Report, 2012.

[24]. Pratt M, Macera CA, Wang G, Higher Direct Medical Costs Associated With Physical Inactivity, The Physician and Sportsmedicine , 2015; 63-70

[25]. Nichols, G, Crow I, Measuring the Impact of Crime Reduction Interventions Involving Sports Activities for Young People, The Howard Journal of Crime and Justice, 2004;43(3): 267-283

[26]. Dobosz RP, Beaty LA, The relationship between athletic participation and high school students' leadership ability, Adolescence. 1999;34(133):215-20.

[27]. Caruso R, Crime and Sport: evidence from Italian Region, The Journal of Socio-Economics, 2011; 40: 455- 463

[28]. Driscoll K, Wood L, Sporting capital : changes and challenges for rural communities in Victoria, Melbourne, Victoria : Centre for Applied Social Research, RMIT, 1999.

[29]. Senduran F, Amman T, Problem-Solving Skills of High School Students Exercising Regularly in Sport Teams, PHYSICAL CULTURE AND SPORT. STUDIES AND RESEARCH, 2015; 42-52.

[30]. Acar K, Akandere M, Bastug G, A study of problem solving skills and non-functional attitudes of children who get training for football. Turkish Journal of Sport and Exercise, 2013; 15(3): 69-74.

[31]. Clark CJ, Problem Solving and Personality Factors of Two At-risk College Populations. Unpublished doctoral dissertation, University of West Virginia, 2002.

[32]. Blumenstein, B, Orbach, I, Psychological Skills in Sport:Training and Application. Happauge, NY: Nova Science. Cox, R.H. (1998). Sport Psychology: Concepts and Applications. Boston: McGraw-Hill. 2010 